\title{
Flipped evaluation: herramientas online para la evaluación participativa $^{*}$
}

\author{
Julia Amorós López ${ }^{1}$, Ana B. Ruescas ${ }^{1}$, Daniel Esperante Pereira ${ }^{1}$, Vicent \\ Girbés-Juan $^{1}$, Roberto Fernandez-Moran ${ }^{1}$, María Moreno Llácer ${ }^{1}$, Adrian \\ Peréz-Suay $^{1}$ y Valero Laparra ${ }^{1}$ \\ ${ }^{1}$ Universitat de València
}

\begin{abstract}
The evaluation of a subject is a fundamental part of the teaching-learning process and one of the main concerns of our students. This is a complex task that requires a lot of effort from the teacher. This is a growing effort in line with the increased weight of continuous evaluation in the current educational system. In this work, different methodologies focused on maximizing the student's performance are presented, thus minimizing the extra effort for the teacher in the evaluation process. We provide several examples of activities throught Moodle platform such as the workshop, glossary, databases, questionnaires, etc. Some of them allow self-assessment once configured, whereas others promote the participation of students in the correction and/or evaluation.
\end{abstract}

Keywords: Evaluation, Moodle tools, questionnaire, workshop.

\begin{abstract}
Resumen
La evaluación de una asignatura es una parte fundamental del proceso de enseñanzaaprendizaje y una de la que más preocupa a nuestros estudiantes. Se trata de una tarea compleja y que requiere un gran esfuerzo por parte del profesor. Un mayor esfuerzo que va parejo al incremento de la evaluación continua, una tendencia en el sistema educativo actual. En este trabajo se presentan diferentes metodologías que maximizan el rendimiento del alumno, tratando a su vez de minimizar el esfuerzo extra por parte del profesor en los procesos de corrección y evaluación. Se proporcionan diversos ejemplos de su uso mediante actividades disponibles en la plataforma Moodle como: taller, glosario, bases de datos, cuestionarios aleatorios, etc. Algunas de estas herramientas permiten la autoevaluación una vez configuradas, en otros casos se presentan metodologías que implican la participación del alumnado en la corrección y/o evaluación.
\end{abstract}

Keywords: Evaluación, herramientas de Moodle, cuestionario, taller.

\footnotetext{
*Proyecto de innovación educativa "Explotación de las herramientas online de la Universitat de València para la evaluación a distancia de asignaturas en el área de ciencia" del curso 2020-21 (UV-SFPIE_PID-1354708)
} 


\section{Introducción}

El proceso educativo presenta importantes desafíos debido a la implantación del modelo educativo de evaluación continua establecido por el Espacio Europeo de Educación Superior. En contraposición con el modelo clásico de clase teórica/magistral y examen/trabajo final, la evaluación continua supone un mayor esfuerzo por parte de los equipos docentes, no siempre resultando en una mayor efectividad a la hora de transmitir conocimientos al alumnado. El enfoque actual de la docencia evidencia la necesidad de implementar cambios metodológicos y de enfoque de la enseñanza. Este cambio de paradigma, que involucra tanto a educadores como a alumnos, debe llevar asociado una serie de procesos de evaluación que permita valorar la consecución de objetivos y destrezas en las distintas materias. En este contexto, las metodologías participativas surgen como un método de trabajo y evaluación que permiten al alumnado identificar sus fortalezas y debilidades, desarrollar un espíritu crítico y comprometerse más con su propio aprendizaje (Rodríguez Casado y Rebolledo Gámez 2017). Esto posibilita una mayor interacción del docente con el alumno, a la vez que reduce la carga de trabajo por parte del profesor. La evaluación por pares aporta un nuevo punto de vista al alumnado y le hace consciente de los objetivos de aprendizaje para realizar esta labor crítica (Florjancic 2020).

En las universidades españolas, la pandemia de COVID-19 ha provocado la suspensión parcial o total de las clases presenciales, impulsando el modo de enseñanza online o híbrida (Abella García y col. 2020), lo que ha ocasionado un auge de las evaluaciones participativas. La búsqueda de medios alternativos a la evaluación tradicional, típicamente exámenes presenciales, ha contribuido a otorgar un mayor peso a la evaluación continua frente a los exámenes finales. En la evaluación continua, es necesario contar con suficientes pruebas que aseguren la adquisición de las competencias de cada materia. Se recomienda (Abella García y col. 2020) que dicha evaluación constituya al menos un $60 \%$ de la nota final, o en el caso de que exista una prueba final, suponga más de un $40 \%$ de la nota. Con el fin de evitar el fraude, la garantía del máximo nivel de identificación de los estudiantes en su modalidad online es un factor clave (Guillén-Gámez y García-Magariño 2015).

En el marco de las metodologías activas en educación superior, el estudiante adquiere mayor protagonismo en su aprendizaje y por ello se exige que, para que la evaluación sea formativa, participe y lleve a cabo también la tarea de evaluación (Arrufat y Rivas 2014). Las herramientas online, como es el caso de Moodle, así como otras metodologías presenciales o virtuales, pueden adaptarse al aprendizaje colaborativo, en el cual profesorado y alumnado intercambian sus roles. Este tipo de aprendizaje, además de fomentar un mayor pensamiento crítico de los estudiantes, favorece su implicación y estimula la discusión. Debe destacarse la importancia que supone la participación e implicación del alumno para conseguir un buen aprendizaje, como así concluye el estudio de Bravo-Agapito y col. 2021, en donde se demostró que el grado de participación de los alumnos en las diferentes actividades de Moodle (glosario, cuestionarios, etc.) pudo predecir la calificación final que el alumno posteriormente obtuvo en la asignatura.

En Cano 2015 se muestra cómo las rúbricas tienen gran utilidad para promover la autorregulación del aprendizaje. La rúbrica es por tanto un aspecto fundamental en la evaluación por pares y/o la autoevaluación en la educación superior, y por tanto, ésta debe reflejar con máxima claridad cómo se evalúa cada una de las tareas (Fernández March 2010), especialmente cuando quienes intervienen en el proceso de evaluación son estudiantes (García-Ros 2011; Alsina y col. 2013). Dicha rúbrica debe tener en cuenta tanto aspectos cuantitativos como cualitativos. De este modo los estudiantes se benefician en el proceso de enseñanza-aprendizaje, ya que conocen de forma explícita las expectativas y criterios de evaluación. Gracias a las rúbricas, los alumnos son capaces de saber qué se va a valorar y cómo se va a evaluar (Sanz 2014). 
Los métodos de evaluación participativa pueden clasificarse en tres bloques:

- Autoevaluación: el estudiante evalúa su acción formativa.

- Co-evaluación: tanto estudiante como profesorado evalúan la acción formativa del estudiante.

- Evaluación por pares: los estudiantes se evalúan entre sí, -dentro de un mismo grupo (intragrupo) o entre grupos distintos (intergrupo)-.

La manera de implementar la evaluación participativa en la evaluación continua de diferentes asignaturas depende en gran medida de la naturaleza de éstas, así como de los recursos disponibles y la propia inventiva del profesor. En el presente caso mostramos métodos y ejemplos puestos en marcha en varias titulaciones de grado de la Universitat de València (tabla 1). Todas se enmarcan en el campo de las STEM (Ciencia, Tecnología, Ingeniería y Matemáticas), algunas más teóricas y otras más experimentales. El número de alumnos de cada asignatura así como las herramientas utilizadas en cada una de ellas se listan en las últimas columnas. Hacer un análisis conjunto de las herramientas y métodos de evaluación propuestos no es tarea fácil. Así, en el presente estudio se presentan diferentes actividades y recursos orientados a las evaluaciones participativas, así como varios ejemplos de su aplicación, resultados y conclusiones de las mismas.

\section{Objetivos}

Objetivo general: Elaborar y contrastar metodologías de evaluación continua minimizando el tiempo requerido por el docente y haciendo partícipe al alumnado de su propio aprendizaje.

Este objetivo general se puede desglosar en los siguientes objetivos:

- Minimizar el esfuerzo que requiere la evaluación continua por parte del profesor en un contexto de clases numerosas y presencialidad decreciente.

- Incentivar el aprendizaje colaborativo y aumentar la participación en las distintas actividades para mejorar la motivación de los estudiantes por la asignatura.

- Reforzar las competencias de los estudiantes de una manera amena mediante metodologías más participativas.

Para ello se utilizarán metodologías que incorporen las nuevas tecnologías mediante el uso de herramientas online propias de la universidad (Moodle) y externas (Socrative, Google Docs, Slides y Forms, CoRubric, Flipped grid):

- Desarrollar metodologías de autocorregibles que ayuden a la autoevaluación del alumno y puedan ser utilizadas para usarse en modelos de evaluación online.

- Desarrollar metodologías de evaluación por pares que fomenten la puesta en común de conocimientos y procuren una evaluación justa.

- Desarrollar metodologías que incluyan recursos tecnológicamente novedosos que resulten atractivos para los alumnos. 
Flipped evaluation: nuevas metodologías de autoevaluación

Tabla 1: Asignaturas de grado analizadas en el estudio de diversas titulaciones.

\begin{tabular}{|c|c|c|c|c|}
\hline Titulación & Asignatura & Curso & № alumnos & Herramientas* \\
\hline Ingeniería Electrónica Industrial & Automatización Industrial (AIND) & $2^{\mathrm{o}}$ & $45-60$ & $\mathrm{C}, \mathrm{BD}, \mathrm{TLL}$ \\
\hline Ingeniería Electrónica Industrial & $\begin{array}{l}\text { Sistemas integrados de Fabricación } \\
\text { (SIF) }\end{array}$ & $3^{\mathrm{o}}$ & $45-60$ & C, TLL, O \\
\hline Ingeniería Electrónica Industrial & Oficina Técnica (OT) & $3^{\mathrm{o}}$ & $45-60$ & C, TLL, O \\
\hline Ingeniería Electrónica Industrial & $\begin{array}{l}\text { Organización y Gestión de la Pro- } \\
\text { ducción (OGP) }\end{array}$ & $3^{\mathrm{o}}$ & $45-60$ & C, TLL, O \\
\hline Ingeniería Electrónica Industrial & $\begin{array}{l}\text { Ingeniería, Sociedad y Universidad } \\
\text { (ISU) }\end{array}$ & $1^{\mathrm{o}}$ & $50-60$ & $\mathrm{O}$ \\
\hline $\begin{array}{l}\text { Ingeniería Electrónica de Teleco- } \\
\text { municación }\end{array}$ & $\begin{array}{l}\text { Ingeniería, Sociedad y Universidad } \\
\text { (ISU) }\end{array}$ & $1^{\mathrm{O}}$ & $50-60$ & $\mathrm{O}$ \\
\hline Física & $\begin{array}{l}\text { Iniciación a la física experimental } \\
\text { (IFE) }\end{array}$ & $1^{\underline{O}}$ & $45-50$ & C, TLL, O \\
\hline Física & $\begin{array}{l}\text { Métodos estadísticos y numéricos } \\
\text { (MEN) }\end{array}$ & $2^{\mathrm{O}}$ & $45-50$ & C, TLL \\
\hline Geografía y Medio Ambiente & Biogeografía (BIO) & $3^{\mathrm{o}}$ & $45-60$ & $\mathrm{C}$ \\
\hline Geografía y Medio Ambiente & Cartografía I (CARTO) & $1^{\mathrm{o}}$ & $80-100$ & $\mathrm{C}$ \\
\hline Maestro/a en Educación Primaria & $\begin{array}{l}\text { Matemáticas para Maestros/as } \\
(\mathrm{MM})\end{array}$ & $2^{\mathrm{O}}$ & $80-100$ & $\mathrm{C}$ \\
\hline Maestro/a en Educación Primaria & $\begin{array}{l}\text { Didáctica de la Aritmética y Reso- } \\
\text { lución de Problemas (DARP) }\end{array}$ & $3^{\mathrm{o}}$ & $80-100$ & $\mathrm{C}$ \\
\hline Ciencia de datos & Señales y sistemas (SyS) & $2^{\mathrm{O}}$ & $60-80$ & $\mathrm{C}, \mathrm{G}$ \\
\hline Ingeniería Telemática & Circuitos electrónicos (CE) & $1^{\underline{o}}$ & $70-80$ & $\mathrm{C}, \mathrm{G}$ \\
\hline
\end{tabular}

* Nota: C: Cuestionarios, BD: Base de datos, G: Glosario, TLL: Taller, O: Exposición oral.

\section{Herramientas de evaluación y su puesta en acción}

A continuación detallamos cuales son las herramientas utilizadas para llevar a cabo los distintos modos de evaluación. La Universitat de València pone a nuestra disposición en Moodle gran cantidad de estas, que conjugadas con recursos externos, nos permite evaluar completamente las diferentes partes de una asignatura: teoría $(\mathrm{T})$, problemas $(\mathrm{P})$, proyectos $(\mathrm{PY})$ y laboratorio $(\mathrm{L})$. 


\subsection{Cuestionarios autocorregibles en Moodle (T, P, L)}

Moodle contiene recursos para la edición de múltiples mecanismos autocorregibles, por ejemplo los cuestionarios. Para ello, es recomendable crear en Moodle un banco de preguntas con cierto grado de flexibilidad, permitiendo estructurar el banco en secciones y subsecciones. Uno de los formatos de preguntas más utilizados es el tipo test con opción múltiple, el cual tiene una gran capacidad en la edición de contenidos e introduce diversos grados de aleatoriedad tanto en la formulación de las preguntas como en las respuestas. El principal escollo que se presenta en la realización de estos cuestionarios es el gran esfuerzo que requiere introducir una batería de preguntas lo suficientemente grande para obtener de ellas el mejor rendimiento. Este rendimiento puede verse mermado por la acción de los alumnos, ya que es posible que estos compartan las preguntas y respuestas entre sí. Para paliar este problema podemos exprimir el uso de los distintos niveles de aleatoriedad presentes en Moodle. La aleatorización, aunque requiere de un esfuerzo extra durante la preparación de las preguntas, permite la utilización de un mismo banco de preguntas en distintas pruebas, incluso dentro del mismo grupo. Aquí analizamos distintos métodos de aleatorización y distintos ejemplos de entornos donde aplicar estos métodos.

Se plantean diferentes niveles de aleatorización: por grupo, pregunta y/o variable:

- La herramienta de creación de grupos de Moodle permite la partición de los miembros de la clase en grupos aleatorios. De esta manera es sencillo implementar de manera virtual el método clásico de generar varios modelos de examen y repartirlos entre los alumnos de forma aleatoria. Además, Moodle permite que sólo si un alumno pertenece a un grupo concreto pueda ver el enlace web al examen. De esta forma, se pueden crear enlaces aparentemente idénticos para cada grupo, conteniendo un examen diferente para cada alumno. Cabe decir que, aunque esta es una manera simple de implementar este método clásico de aleatorización, su contrapartida es la necesidad de generar diferentes exámenes y su posterior corrección.

- La herramienta de aleatorización por preguntas permite crear cuestionarios que seleccionen preguntas del banco de manera aleatoria.

- La herramienta de aleatorización de variables dentro de preguntas resulta extremadamente útil para la creación de exámenes de evaluación online y para diversas aplicaciones que veremos más adelante. El objetivo de esta herramienta es la creación de preguntas en las que los datos de entrada son generados de forma aleatoria y las soluciones se calculan automáticamente con Moodle.

Actualmente, en Moodle disponemos de dos opciones principales de preguntas calculadas que nos permiten aleatorizar las variables dentro de la pregunta.

- La primera opción es la pregunta calculada y calculada simple. En estas preguntas se requiere introducir como respuesta un valor numérico. Además, ambas permiten la posibilidad de introducir un margen de error en la respuesta.

- La segunda opción es la pregunta de opción múltiple calculada. A diferencia de las versiones calculadas, esta modalidad ofrece al estudiante diferentes opciones de respuesta. Con esta opción se permite el cálculo automático del resultado a partir de fórmulas propuestas por el editor, que puede depender de las variables de entrada.

Aquí presentamos distintas opciones para sacar partido de una banco de preguntas aleatorizado: 
- Evaluación online (T) y evaluación continua (P, L). Dado el incremento de la docencia online, es imprescindible desarrollar metodologías que aseguren en la medida de lo posible una evaluación online justa. Un punto clave es la capacidad de aleatorizar las pruebas para que cada alumno sea evaluado mediante una prueba independiente y única, pero garantizando que el nivel sea similar al de sus compañeros. En este entorno se propone combinar la aleatorización por pregunta y la aleatorización de las variables vistas anteriormente. De este modo un examen de 10 preguntas que incluya dos versiones diferentes de la misma pregunta tendría $2^{10}=1024$ versiones distintas. Además si cada pregunta está implementada con variables aleatorias, no solo los exámenes tendrán versiones de preguntas distintas sino que cada pregunta tendrá valores distintos para las variables. Esto limita la posibilidad de compartir las respuestas, y además, si el alumno dispone de un tiempo ajustado para la realización del examen, se dificulta que un alumno pueda ayudar a realizar otros exámenes además del suyo. En la sección 4.1 veremos un ejemplo práctico. Esta técnica se puede adaptar para la creación de cuestionarios de evaluación continua donde se incluyan una o dos preguntas de examen del banco de preguntas con variables aleatorizadas. De este modo se aprovecha el banco de preguntas de la asignatura de manera múltiple. La aleatorización de las variables asegura que si bien un modelo de pregunta puede aparecer en un cuestionario y en un examen, las variables de esa pregunta serán diferentes, lo cual obliga al alumnado a aprender a resolver el ejercicio sin memorización previa de las respuestas. En la sección 4.1 se presenta también otro ejemplo práctico.

- Auto-aprendizaje (P). Si el banco de preguntas realizado es lo suficientemente extenso, la aleatorización se puede utilizar como herramienta de creación de pruebas para autoevaluación. Es decir, el alumno tendrá acceso antes del examen a un cuestionario con formato similar (o igual) al del examen que se realizará en la prueba oficial, de modo que pueda practicar y autoevaluarse antes de presentarse al examen oficial. El número de preguntas necesarias dependerá de la asignatura. Nótese que la progresión de la cantidad de exámenes diferentes es geométrica. Pongamos como ejemplo 2 exámenes oficiales al año (uno por cada convocatoria) y 10 preguntas por examen. Al inicio del tercer año en el que se imparte la asignatura, se tendrían más de un millón de versiones de exámenes distintos $\left(4^{10}\right)$, de los cuales se pueden obtener infinidad de versiones con variables distintas. El único esfuerzo extra requerido es la introducción de las preguntas de examen en la plataforma, que para este ejemplo particular serían 40 preguntas en el periodo de 2 años.

Las preguntas calculadas introducen variables con valores generados de forma aleatoria de modo que permite un alto grado de reutilización de las preguntas.

\subsection{Base de datos de problemas $(\mathrm{P})$}

Esta herramienta permite que los alumnos resuelvan unos ejercicios, bien de un boletín o incluso propuestos por ellos, y que después los pongan a disposición de sus compañeros en el Aula Virtual $(\mathrm{AV})$. De esta forma, se fomenta el aprendizaje colaborativo entre los estudiantes y que tengan que esforzarse en seguir una metodología de resolución, ya que el resto de compañeros deberán comprender la solución propuesta. Esto se evalúa dando un peso a la presentación, fomentando que se realicen incluso en $\mathrm{LTT}_{\mathrm{EX}}$ o que puedan presentar un vídeo explicativo con la resolución del ejercicio. Además, se intentará usar herramientas que permitan que los estudiantes puedan 
corregirse o poner comentarios entre ellos. Dentro del AV, se ha implementado esta base de datos de problemas de dos maneras distintas:

- Base de datos de Moodle: permite definir diferentes tipos de entradas o fichas, que previamente debemos de configurar. Se seleccionan los campos que tendrá cada una de las entradas y después los alumnos añadirán cada ejercicio como una entrada nueva. Los alumnos pueden editar y añadir comentarios en las entradas del resto de compañeros.

- Glosario de problemas: un glosario normalmente se utiliza para crear una lista de términos de la asignatura. En este caso, aprovechando que en el glosario se pueden añadir adjuntos e imágenes a las entradas, los estudiantes añadirán sus ejercicios resueltos como una entrada nueva. Además permite realizar comentarios a cada entrada.

Estas herramientas se han utilizado con éxito en diversas asignaturas para resolver problemas propuestos o incluso para que propongan problemas inventados. En la sección 4.2 se proporcionan más detalles de su aplicación práctica.

Destacar que la herramienta base de datos es bastante más compleja de utilizar que el glosario, ya que se debe configurar una plantilla o ficha con los campos requeridos, y que después los alumnos deben completarla cada vez que añaden una entrada.

\subsection{Taller de Moodle (P, PY)}

Esta actividad permite la autoevaluación y la evaluación por pares de trabajos. Está dividida en cinco fases temporales: configuración, envío, evaluación, cálculo de calificaciones y cierre (ver figura 1). En la fase de envío, los alumnos envían su tarea o proyecto, y en la fase de evaluación deberán evaluar a sus compañeros en función de unos aspectos o criterios configurados por el profesor. Se permite el uso de escalas, rangos y campos de texto para cada uno de los aspectos. Las tareas pueden enviarse de forma individual o en grupos, y la asignación de las evaluaciones puede ser realizada por el profesor manualmente o de forma automática. En la fase final, los alumnos tienen acceso a las evaluaciones de sus trabajos por parte de sus compañeros, pudiendo recibir una respuesta mucho más rápida que si es únicamente el profesor el que debe de corregir un gran volumen de trabajos o proyectos. Destacar que como en esta actividad se realiza la evaluación por pares, es necesario definir una rúbrica adecuada y clara para los alumnos. En la sección 4.3 se describe con más detalle algunos ejemplos de aplicación del taller y sus resultados.

\subsection{Aprendizaje participativo con exposición oral y recursos multimedia (L, PY)}

En diversas asignaturas se realizan exposiciones orales de los estudiantes sobre el temario o para explicar un proyecto. Además, estas exposiciones se pueden grabar en vídeo para evaluarlas posteriormente, tanto por los estudiantes como por el profesor. La Universitat de València ofrece repositorios para el almacenamiento de este material multimedia de manera que está accesible al alumnado y docentes. En la sección 4.4 se proporcionan detalles de su aplicación en algunas asignaturas.

Al igual que la actividad taller, con el objetivo de homogeneizar los criterios de evaluación de las exposiciones orales, en las diferentes asignaturas se crearon rúbricas que tenían que seguir todas las partes. Es por ello, que en la siguiente sección se justifica su necesidad y se proporcionan herramientas para definir estas rúbricas. 


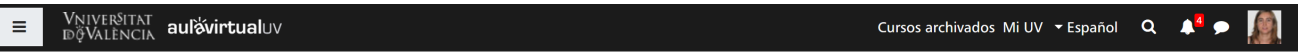

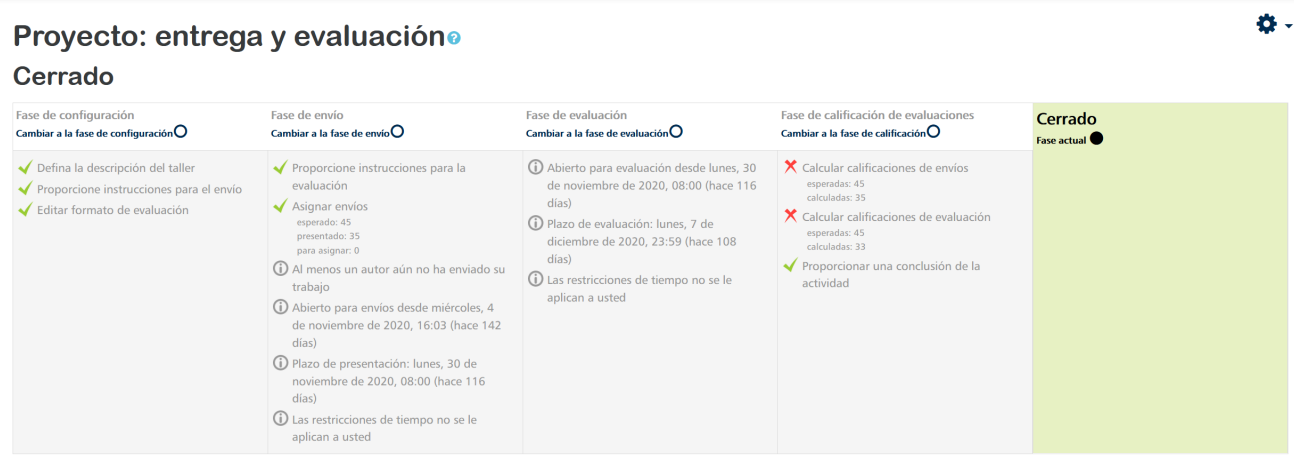

Fig. 1: Ejemplo de las diferentes fases de un taller.

\subsection{Rúbricas de evaluación ( $\mathrm{T}, \mathrm{L}, \mathrm{P}, \mathrm{PY})$}

El uso de rúbricas por parte del profesor para la evaluación de la teoría y problemas de la asignatura es ampliamente utilizado, y muy recomendable en el caso de los laboratorios impartidos por diversos profesores. Sin embargo, en este estudio nos centraremos en las rúbricas desarrolladas para la autoevaluación o la evaluación por pares para las asignaturas con exposiciones orales y/o basadas en el aprendizaje por proyectos, donde se suele utilizar la herramienta taller. Esta evaluación implica una gran responsabilidad y compromiso por parte del estudiante (Arrufat y Rivas 2014), y pese a la posibilidad de que los estudiantes no sean objetivos en su autoevaluación o cuando realizan una evaluación recíproca, la experiencia ha demostrado que tienden a ser bastante críticos e intentan realizar una evaluación justa. En Valverde Berrocoso y col. 2014 se muestran las potencialidades de las rúbricas para la autorregulación y autoeficacia del estudiante, la calidad de la evaluación y la mejora de la docencia, pero según los autores es imprescindible elaborar rúbricas que cumplan con criterios de fiabilidad y validez.

Existen numerosas plataformas para realizar e-rúbricas en el contexto de docencia online. Entre ellas destacan las siguientes: iRubric, RubiStar, CoRubrics y Moodle. La plataforma iRubric es una herramienta integral de desarrollo, evaluación y uso compartido de rúbricas iRubric. RubiStar es una herramienta gratuita para ayudar a los profesores a crear rúbricas de calidad que además incluye una gran cantidad de ejemplos que los docentes comparten en su web RubiStar. Otra herramienta bastante extendida es CoRubrics, un complemento para hojas de cálculo de Google que permite realizar un proceso completo de evaluación con rúbricas CoRubrics. Por último, Moodle también incorpora una funcionalidad para realizar rúbricas y además existe mucha documentación sobre la elaboración de rúbricas con esta herramienta. 


\section{Resultados}

En esta sección se analizan los resultados obtenidos al utilizar las herramientas propuestas en la sección 3, con ejemplos concretos de las asignaturas que se han introducido en la tabla 1. En cada apartado se indica la herramienta y el uso que se le ha dado y que se está analizando en cada caso.

\subsection{Análisis del uso de cuestionarios autocorregibles}

Los cuestionarios se han utilizado en muchas de las asignaturas, tal y como se muestra en la tabla 1. En algunas con más contenido teórico como BIO, CARTO, AIND, SIF, OT y OGP, se han utilizado cuestionarios con selección y aleatorización de las preguntas en diferentes subcategorías, así como el uso de diferentes grupos aleatorios. En estos casos se requiere un número de preguntas elevado, ya que cada pregunta del banco de preguntas es única y no depende de ninguna variable.

La aplicación basada en los mecanismos de cuestionarios autocorregibles de la sección 3.1 con aleatorización de variables se ha utilizado con éxito en las asignaturas IFE, $M E N, M M, S y S$ y $C E$.

Como caso particular analizamos en más detalle la asignatura de $C E$, cuya evaluación se realizó online debido a la pandemia del Covid-19. Para ello analizamos la diferencia de notas entre el curso 2018-19, donde el examen se hizo presencial, y el curso 2019-20, donde el examen se hizo online. Los resultados sobre [80-100] alumnos se pueden ver en la tabla 2. Como se puede comprobar, aunque existe una diferencia apreciable en la cantidad de alumnos que se presentaron a la convocatoria, la diferencia no hace pensar que haya habido un proceso de falseo de exámenes generalizado. Nótese que el porcentaje de suspensos es alrededor del $20 \%$ durante la evaluación online, respecto al $30 \%$ del año anterior. Esto hace pensar que, aunque el método de evaluación online no es el ideal, puede ser aplicable. En cualquier caso, estos resultados deben ser contrastados con los del curso 2020-21, en el cual el examen presencial será creado utilizando el mecanismo de generación automática.

Tabla 2: Resultados de exámenes teóricos de la asignatura de CE.

\begin{tabular}{lllll}
\hline Curso & No presentados & Suspensos & Aprobados & Notable \\
\hline $2018-19$ (presencial) & $32.8 \%$ & $31.4 \%$ & $34.3 \%$ & $1.4 \%$ \\
\hline $2019-20$ (on-line) & $13.22 \%$ & $19.0 \%$ & $55.4 \%$ & $12.4 \%$ \\
\hline
\end{tabular}

En este segundo experimento mostramos el uso de las preguntas calculadas, las cuales permiten aumentar la diversidad de las cuestiones sin incrementar drásticamente el trabajo de preparación y corrección.

Para ello se ha realizado un ejercicio práctico mediante una pregunta calculada en los cuestionarios habituales de autoevaluación que se realizan al finalizar cada tema en la asignatura de $C E$. En la figura 2 se puede ver un ejemplo de las preguntas realizadas. En este caso los valores de la resistencia (R1), la bobina (L1) y el condensador (C) son inicializados aleatoriamente por la plataforma. Para poder ser mas equitativos en las correcciones se le pide al alumno que entregue, a través de una tarea específica de Moodle, la resolución del ejercicio hecha a mano. De este modo se puede comprobar que la respuesta no fue aleatoria y además permite al alumno consultar los fallos en el ejercicio. 
Aunque la revisión de la resolución del problema supone una carga extra para el profesor, la herramienta posibilita tener una observación automática del nivel de cada uno de los alumnos, que de otro modo debería realizarse manualmente. Además, reduce el riesgo de plagio, al permitir ejercicios con distintos valores de entrada a cada alumno.

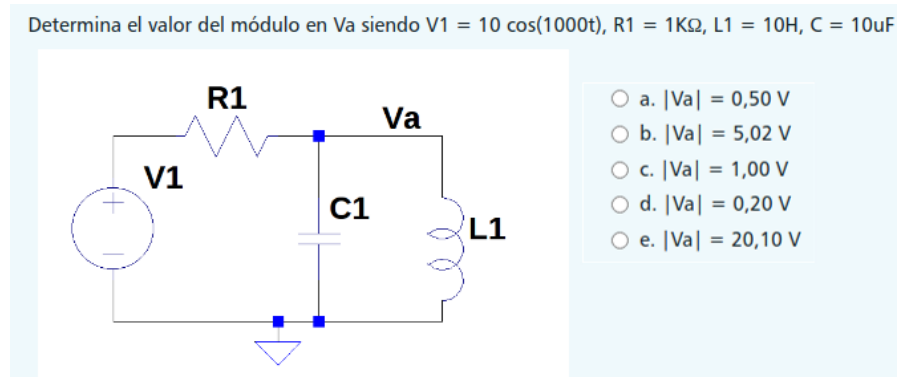

Fig. 2: Ejemplo de pregunta autocalculada incluida en los cuestionarios.

En la figura 3, podemos observar la diferencia de notas entre el curso en el cual las preguntas únicamente fueron teóricas y el curso donde se ha introducido la pregunta práctica. Las notas claramente son más bajas en el segundo caso, lo cual puede deberse a que las respuestas a las preguntas de teoría eran fácilmente obtenibles de sus apuntes, mientras que la resolución del ejercicio requería de un trabajo previo de comprensión. Estos resultados se complementarán al final del presente curso con una comparativa de notas finales respecto a años anteriores.

Cuestionario 1

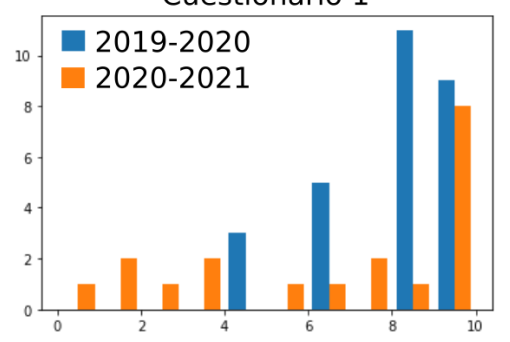

Cuestionario 2

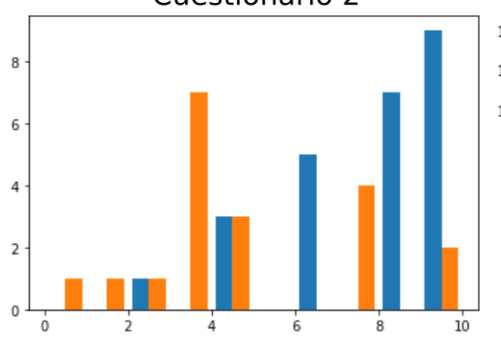

Cuestionario 3

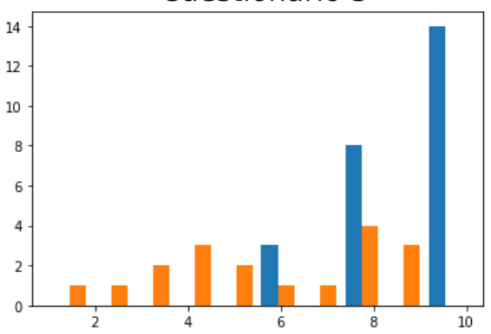

Fig. 3: Histograma de notas para cuestionarios sin incluir preguntas calculadas (2019-2020) e incluyendo preguntas calculadas (2020-2021).

\subsection{Análisis del uso de bases de datos de problemas}

En las asignaturas de $C E$ y $S y S$ se ha utilizado la herramienta del glosario para fomentar la resolución de ejercicios por parte del alumnado. Con esta herramienta de Moodle, se insta a los alumnos a que suban la solución de una serie de problemas previamente seleccionados por el docente. El docente tiene dos versiones de los mismos problemas "sin soluciones" y "con soluciones". Primero se permite el acceso a la serie de problemas sin soluciones y se insta a los alumnos a que incluyan sus propias soluciones. De este modo, el primer alumno que proporcione la solución obtendrá una nota extra de evaluación continua. Se tiene en cuenta además que la presentación sea 
la adecuada, para que así dichos problemas resueltos puedan ser utilizados por otros estudiantes. Por otro lado, la identificación de errores en los ejercicios de otros compañeros también es puntuable. Al finalizar el trimestre, se presentan los ejercicios con soluciones de forma que los estudiantes se puedan autocorregir.

En particular, en el curso 2019-2020, el 39,5\% de los estudiantes presentó algún ejercicio y el $78 \%$ de los ejercicios quedaron resueltos por los alumnos de la asignatura de $C E$. En la asignatura de SyS, para el curso 2020-2021, el 51,2\% de los alumno presentó algún ejercicio, siendo el 92,1\% de los ejercicios resueltos por alumnos. También se obtuvieron ejercicios resueltos en vídeo, los cuales podrán ser utilizados en años posteriores.

En la asignatura $A I N D$ se ha utilizado la herramienta base de datos tanto para resolver problemas de un boletín como para que los alumnos propongan ejercicios inventados por ellos en el curso 2020-2021. El $63 \%$ de los alumnos subió algún ejercicio y el $53 \%$ comentó las soluciones de otro compañero.

El alto grado de participación en todas las asignaturas donde se han utilizado estas herramientas de base de datos o glosario, muestra que es un recurso muy potente para el aprendizaje colaborativo y que permite aumentar la interacción entre los alumnos dentro del AV, lo que permite además que se pueda cuantificar en la evaluación continua.

\subsection{Análisis del uso de taller para evaluación continua}

El taller se ha utilizado con éxito en las asignaturas de AIND, SIF, OT y OGP para evaluar proyectos presentados por los alumnos, tanto de forma individual como en grupos. Estas asignaturas incluyen la realización de un proyecto que van entregando en diferentes fases (no confundir con las fases del taller), donde se realizan seguimientos intermedios en los que la actividad del taller se utiliza para que los propios alumnos evalúen a sus compañeros, lo cual ayuda a detectar errores de forma temprana, mejora la calidad final de los proyectos y ayuda en el seguimiento de la asignatura por parte de los alumnos.

Como se ha indicado antes, al tener que evaluarse entre ellos, cobra especial importancia la definición de una rúbrica de evaluación, que debe ser clara, sencilla y que defina cuidadosamente los requisitos y aspectos más importantes.

Concretamente, en la asignatura $A I N D$ se ha utilizado la herramienta taller para co-evaluar un proyecto individual de automatización en el curso 2020-2021. El $75 \%$ del alumnado participó en la actividad evaluando dos trabajos, y la correlación entre las notas de la profesora y los alumnos fue de un $79 \%$. Cabe destacar que la co-evaluación ayuda al profesorado en la corrección de los trabajos, ya que aunque muchas de las valoraciones coinciden, suelen aporta otros puntos de vista.

En las asignaturas de $S I F$ y $O G P$ también se está utilizando esta actividad para realizar el seguimiento de las diferentes fases de entrega de un proyecto y su evaluación por pares por parte de los alumnos. El curso anterior se realizaban mediante tareas y correos particulares con los enlaces a los proyectos y vídeos de presentación. Además de las ventajas indicadas en el caso anterior, el uso del taller está facilitando la gestión y organización de este tipo de actividades para el profesorado. Destacar también que al disponer de una evaluación inicial del alumnado, esta puede utilizarse para detectar y priorizar la corrección de aquellos trabajos más deficientes para proporcionar una rápida respuesta al alumno antes de la fase de entrega siguiente. 
En la asignatura de $S y S$ se ha utilizado la herramienta de taller para definir una actividad al final del curso donde los alumnos han de proponer una pregunta que ellos consideren que es de nivel de examen, además de proporcionar su respuesta. Cada estudiante acababa evaluando a dos compañeros (evaluación por pares).

Los resultados son claramente beneficiosos si se atiende a dos aspectos. Por un lado, los estudiantes desarrollan diferentes habilidades, ya que deben hacer un resumen de la asignatura valorando las partes mas importantes, han de aprender a resolver una pregunta que ellos consideran de nivel de examen, así como presentar de forma didáctica los resultados; además, han de analizar los resultados de otros compañeros, resolviendo por tanto dos preguntas de nivel de examen. En segundo lugar, el docente solo tiene que controlar que las evaluaciones realizadas por los alumnos se ajustan a la realidad. Se obtiene una visión bastante completa de lo que los alumnos consideran el nivel de una pregunta de examen y de cual es su capacidad para resolverlas. Además, el docente obtiene una batería de preguntas de nivel de examen corregidas y presentables.

En el caso particular de la asignatura de SyS, en el curso 2019-2020, un $85 \%$ de los alumnos participaron en la actividad obteniendo una evaluación por sus compañeros con una media de 8,3 y en el curso 2020-2021 un 87,5\% de los alumnos participaron en la actividad obteniendo una evaluación por sus compañeros con una media de 8,1. Además, un total de 25 preguntas (el 28,3\%) del curso 2019-2020 fueron usadas como ejemplo para los alumnos del curso 2020-2021.

\subsection{Análisis del aprendizaje participativo con exposición oral y recursos multimedia}

En la asignatura $I S U$, una de las actividades propuesta a los alumnos consistía en la preparación de un tema, su presentación oral en el aula y la grabación en vídeo para evaluarlo posteriormente, tanto por ellos mismos como por el profesor (co-evaluación). La autoevaluación por parte de los estudiantes de su propio vídeo es una parte importante de su aprendizaje, ya que normalmente son bastante críticos consigo mismos.

Una estrategia similar se emplea en la asignatura de $I F E$ en la que los estudiantes exponen una de las prácticas realizadas en el laboratorio, incluyendo objetivos, dispositivo experimental y explicación de los resultados obtenidos.

El porcentaje de participación en esta actividad en $I S U$ fue del $75 \%$ del total del alumnado matriculado. La figura 4 muestra la distribución de notas de los alumnos en un rango de 0-100 para los tres tipos de evaluación (profesor, autoevaluación y los pares). Puede observarse que los estudiantes son bastante críticos con sus compañeros y consigo mismos, ya que la media de la distribución de notas es de hecho algo inferior a la asignada por el profesor. Una posible explicación es que los alumnos han seguido la rúbrica de forma estricta, mientras que el profesorado puede haber tenido en cuenta otros aspectos de forma global. La evaluación consistía en realizar una descripción de la valoración de la rúbrica en formato texto libre y asignar una calificación numérica. Cabe destacar que, mientras que la práctica totalidad del alumnado redactó el informe en texto libre, esto no fue así para la calificación numérica: sólo un $18 \%$ de ellos se autoevaluó numéricamente y un $74 \%$ puntuó a sus compañeros. Aunque claramente la participación y evaluación colectiva en la tarea ha sido muy exitosa, de cara a obtener una valoración cuantitativa de la nota por parte de los alumnos conviene utilizar herramientas que les obliguen a introducir una calificación numérica o de lo contrario la tarea no se considera realizada. Ejemplo de ellos podrían ser las herramientas de taller y las de rúbricas previamente mencionadas. Para la asignatura $I F E$ se ha tenido en cuenta este aspecto. 


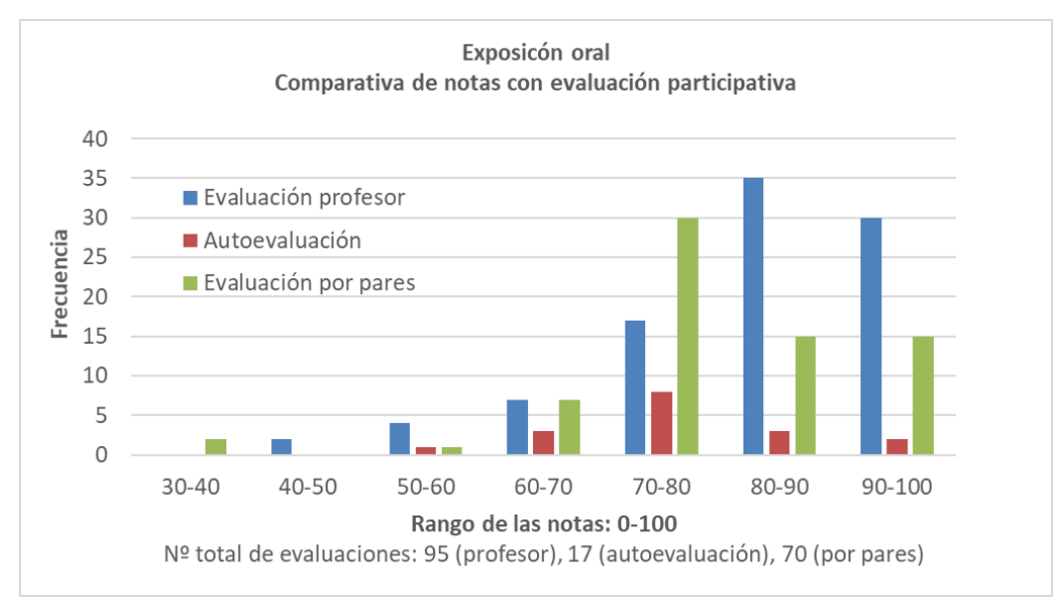

Fig. 4: Comparativa de las notas numéricas en la evaluación participativa de exposición oral en la asignatura ISU.

También se utiliza la exposición oral en las asignaturas $O T, O G P$ y $S I F$, en las que los alumnos realizan un proyecto en grupo que se debe presentar en clase en formato PechaKucha (20x20: 20 diapositivas, 20 segundos cada una, Beyer 2011). Así se pueden exponer todos los trabajos en poco tiempo y de una forma mucho más dinámica y vistosa que con el formato tradicional.

En todas estas asignaturas, estas presentaciones inicialmente se realizaban en clase de forma presencial con una rúbrica dada, lo cual permitía además controlar la asistencia de los estudiantes a todas las presentaciones. Sin embargo, el curso pasado se adaptaron para realizarse de forma no presencial debido al Covid-19 mediante la grabación de un vídeo y su posterior evaluación.

\subsection{Análisis del uso de rúbricas para evaluación continua}

Como se ha comentado anteriormente, el uso de las rúbricas se ha utilizado en todas las asignaturas, pero nos gustaría destacar aquí su uso tanto para la evaluación continua de trabajos y/o proyectos como para la exposición oral de estos en ISU, OT, OGP, AIND, SIF, BIO y SyS.

Una de las principales ventajas de utilizar la herramienta de rúbricas de Moodle es que se puede integrar en la evaluación de diferentes actividades dentro de la plataforma y permite la importación y exportación de las rúbricas diseñadas para su reutilización en otros entornos y/o actividades.

En el caso concreto de las asignaturas $O T$ y $O G P$, los resultados muestran que la evaluación continua tiende a incrementar la nota del alumnado con respecto a la del examen. Concretamente, en una escala de puntuación de 0 a 10, se obtuvo una nota cuyo valor medio era de 1, 42 puntos superior en la evaluación continua a la del examen final, con una desviación típica de 1,4 puntos. Además, se observó una correlación directa entre la nota obtenida por el alumnado durante la evaluación continua aplicando rúbricas y la nota del examen final. Esto no ocurría en cursos anteriores en los que no había evaluación continua en el desarrollo de los trabajos. 
Se han analizado y validado herramientas para evaluar diferentes actividades formativas: teoría, problemas, proyectos y laboratorio. Los resultados demuestran que las metodologías de evaluación activa son justas y reducen el tiempo de dedicación del docente.

\section{Conclusiones}

El incremento de peso en la evaluación continua obliga a desarrollar metodologías que minimicen el trabajo extra que debe realizar el docente. En el presente artículo hemos presentado las herramientas utilizadas en diferentes disciplinas científicas, tratando de establecer sus principales características y su forma habitual de uso, con ejemplos específicos en las diferentes asignaturas. Se debe destacar la gran variedad de disciplinas así como la modalidad de docencia, ya sea mediante clases teóricas, prácticas y de laboratorio.

Entre las herramientas de evaluación hemos utilizado con profusión la versatilidad de los cuestionarios, destacando sus posibilidades de aleatorización, y sus diferentes modalidades de evaluación. Las bases de datos de problemas y los glosarios constituyen un recurso que facilita el aprendizaje colaborativo. Los talleres requieren de un esfuerzo adicional de preparación y se desarrollan de una manera más dilatada en el tiempo, pero suponen un elemento de control del desarrollo de los trabajos tanto para el docente como para los propios alumnos. La exposición oral, quizás más tradicional, se moderniza con la introducción de la evaluación de vídeos que quedan en un repositorio para su evaluación posterior en la modalidad que se requiera. Muchas de las herramientas mencionadas fundamentan la evaluación en las rúbricas, por lo que se han destacado de manera independiente pese a constituir un elemento transversal.

Los resultados presentados no se han analizado de manera global, dada la diversidad de las materias donde se han aplicado los métodos de evaluación presentados, pero podría destacarse que la participación del estudiantado es relativamente alta, aunque variable: desde cerca del $50 \%-75 \%$ de participación con las bases de datos de problemas, el $75 \%$ de la exposición oral y recursos multimedia, a casi el $90 \%$ en los talleres y en los cuestionarios.

Dos hechos que podrían destacarse tras este análisis son que, aunque algunos de los métodos presentados requieren de cierto esfuerzo de preparación por parte del docente, a la larga suponen un ahorro de tiempo y ayudan al profesor a identificar las fortalezas y debilidades de su pedagogía docente. El segundo hecho es que estas herramientas no sólo son útiles para evaluar, sino que son ventajosas para desarrollar otras competencias más globales: lingüística, tecnológica, resolver problemas bajo presión (tiempo limitado) o la realización de un análisis más profundo de las materias. De este modo, el alumno no debe tratar sólo de memorizar, sino también analizar, aplicar, desarrollar y evaluar la calidad de lo aprendido, aspectos fundamentales en la nueva era de la información.

Las propuestas que se muestran en el presente estudio pretenden ofrecer nuevas perspectivas y herramientas concretas y útiles para que los docentes puedan adaptarse a la nueva realidad digital en la enseñanza, acelerada sin duda por la pandemia del Covid-19, a la vez que los alumnos puedan adquirir nuevas competencias en línea con los cambios que la sociedad demanda. 


\section{Referencias bibliográficas}

Abella García, V. y col. (2020). "Guía de recomendaciones para la evaluación online de las universidades públicas de Castilla y León”. En: pág. 30.

Alsina, Pep y col. (2013). "Rúbricas para la evaluación de competencias". En: Universitat de Barcelona. Institut de Cičncies de l'Educació \& Ediciones.

Arrufat, M.J. Gallego y M. Raposo Rivas (2014). "Compromiso del estudiante y percepción del proceso evaluador basado en rúbricas". En: REDU: Revista de Docencia Universitaria 12.1, pág. 197.

Beyer, Alisa Miller (2011). "Improving student presentations: Pecha Kucha and just plain PowerPoint". En: Teaching of Psychology 38.2, págs. 122-126.

Bravo-Agapito, J. y col. (2021). "Early prediction of undergraduate Student's academic performance in completely online learning: A five-year study". En: Computers in Human Behavior 115.

Cano, Elena (2015). "Las rúbricas como instrumento de evaluación de competencias en educación superior:¿ uso o abuso?" En: Revista de currıculum y formación de profesorado 19.2, págs. $265-280$.

Fernández March, A. (2010). "La evaluación orientada al aprendizaje en un modelo de formación por competencias en la educación universitaria". En: Revista de Docencia Universitaria 8.1, págs. 11-34.

Florjancic, V. (2020). "Peer assessment among business students". En: International Journal of Learning Technology 15.1, págs. 4-25.

García-Ros, R. (2011). "Análisis y validación de una rúbrica para evaluar habilidades de presentación oral en contextos universitarios". En: Electronic Journal of Research in Educational Psychology 9.3, págs. 1043-2062.

Guillén-Gámez, F. D. e I. García-Magariño (2015). "A technique for designing glossary activities with facial authentication". En: Journal of E-Learning and Knowledge Society 11.1, págs. $125-138$.

Rodríguez Casado, M.R. y T. Rebolledo Gámez (2017). "Evaluación de metodologías participativas: una experiencia en el ámbito universitario". En: Revista de Humanidades 31, págs. 99-121.

Sanz, M.P. Garcia (2014). "La evaluación de competencias en Educación Superior mediante rúbricas: un caso práctico". En: Revista electrónica interuniversitaria de formación del profesorado 17.1, págs. 87-106.

Valverde Berrocoso, Jesús y col. (2014). "El uso de e-rúbricas para la evaluación de competencias en estudiantes universitarios. Estudio sobre fiabilidad del instrumento". En: REDU. Revista de docencia universitaria 12.1, págs. 49-79. 